\title{
HIV Status
}

National Cancer Institute

\section{Source}

National Cancer Institute. HIV Status. NCI Thesaurus. Code C157155.

The result of testing to determine if an individual is infected with the human immunodeficiency virus. 